\title{
RESULTADOS DE LA COMPARACIÓN INTERLABORATORIOS DE CALIBRACIÓN DE MULTÍMETROS CAMET 2009
}

\section{Resumen}

Se presentan los resultados de la segunda comparación en calibración de multímetros, organizada en Costa Rica, por el Laboratorio Metrológico de Variables Eléctricas (LMVE) en la que participaron doce laboratorios, con capacidad para calibrar multímetros digitales. El LMVE es un laboratorio del Instituto Costarricense de Electricidad (ICE) y que ha sido designado como laboratorio nacional en electricidad, magnetismo, tiempo y frecuencia por el Laboratorio Costarricense de Metrología (LACOMET)

La comparación siguió los lineamientos de la INTE ISO/IEC Guía 43-1:2000 Ensayo de aptitud por comparaciones interlaboratorios. Parte 1: Desarrollo y funcionamiento de programas de ensayos de aptitud (INTECO, 2000) y se analizaron tres mensurados, a saber: corriente, voltaje y resistencia.

Se tuvo participación de laboratorios nacionales y el laboratorio nacional de Panamá, evidenciando la importancia de este tipo de ejercicios, los cuales pueden ampliarse, por ejemplo, hacia la región Centroamericana.

Entre los principales resultados están el buen uso que puedan hacer los laboratorios participantes para implantar acciones de mejora en su gestión metrológica, así como el aumento en el conocimiento a nivel nacional de la capacidad de medición en estas magnitudes, de voltaje, corriente, resistencia entre otros.

Palabras clave: multímetro, calibración, comparación, interlaboratorios, metrología.

\begin{abstract}
Results are presented for the second multimeter calibration intercomparison organized in Costa Rica by Laboratorio Metrológico de Variables Eléctricas (LMVE), with participation of twelve laboratories with capability for calibration of multimeters. LMVE belongs to Instituto Costarricense de Electricidad and is designated by Laboratorio Costarricense de Metrología (LACOMET) as national laboratory in electricity, magnetism, time and frequency.

The comparison followed guidance in (Costa Rican version of) ISO/IEC Guide 43-1 Proficiency testing by interlaboratory comparisons -- Part 1: Development and operation of proficiency testing schemes, analyzing three measurands: current, voltage and resistance.

There was participation of Costa Rican laboratories and the Panama national laboratory, showing the importance of these exercises, which can be expanded to the Central American region.

Among the main results are the use by participant laboratories for improvement action in there metrology activities, and better national knowledge in measuring voltage, current and resistance.
\end{abstract}

Keywords: multimeter, calibration, comparison, interlaboratory, metrology.

Recibido: 09 de febrero del 2010 - Aprobado: 11 de febrero del 2010.

\section{INTRODUCCIÓN}

El Laboratorio Metrológico de Variables Eléctricas (LMVE) del ICE, como laboratorio designado por LACOMET en Electricidad e Infocomunicaciones, de acuerdo con el convenio firmado por LACOMET y el ICE en setiembre de 2004, organizó una comparación de calibración de multímetros.

LanormaINTE-ISO/IEC 17025:2005 Requisitos generales para la competencia de laboratorios de ensayo y calibración (INTECO, 2005) incluye: 
"5.9 Aseguramiento de la calidad de los resultados de ensayo y calibración

El laboratorio debe tener procedimientos de control de calidad para dar seguimiento a la validez de los ensayos y las calibraciones que realiza. Los datos resultantes deben ser registrados de tal manera que se puedan detectar tendencias y, cuando sea factible, se deben aplicar técnicas estadísticas para la revisión de los resultados. Este seguimiento debe ser planificado y revisado y puede incluir, pero no estar limitado a, lo siguiente:

a) uso regular de materiales de referencia certificados y/o controles de calidad internos utilizando materiales de referencia secundarios;

b) participaciónenprogramasdecomparaciones interlaboratorios o ensayos de aptitud;

c) repetición de ensayos o calibraciones utilizando el mismo o diferentes métodos;

d) reensayo o recalibración de los objetos retenidos;

e) correlación de resultados para diferentes características del objeto."

La política "ECA-MC-P17 Política y criterios para la participación en ensayos/ pruebas de aptitud y otras comparaciones para los laboratorios" incluye, entre algunas formas de aseguramiento de la calidad:

\section{“7. REQUISITOS GENERALES}

Los criterios del ECA para la Acreditación de laboratorios establecen los siguientes requisitos en relación a la participación en ensayos/pruebas de aptitud y otras comparaciones:

7.1. Demostración de la competencia técnica de los laboratorios para la realización de los ensayos o calibraciones acreditados o en proceso, por medio de la participación en programas de ensayos/pruebas de aptitud y otras comparaciones para los laboratorios de ensayo y de calibración, donde tales actividades estuvieren disponibles.
7.2. Una cantidad mínima de ensayos/ pruebas de aptitud y otras comparaciones adecuadas para los laboratorios es:

7.2.1. Una participación antes de la Acreditación,

7.2.2. Para el caso de los laboratorios de ensayo deberán participar como mínimo una vez cada cuatro años en ensayos/ pruebas de aptitud u otras comparaciones relacionadas con cada disciplina principal correspondiente al alcance de la Acreditación, ejemplos de disciplinas se dan en la nota 3.

Nota 3: Ejemplos de disciplinas:

- Análisis ambiental: suelo (incluyendo desechos sólidos, lodo, compost, foliares);

- Análisis ambiental: aguas (aguas residuales, aguas subterráneas, agua potable);

- Análisis ambiental: aire (emisiones e inmisiones);

- Pruebas de materiales: parámetros químicos

- Pruebas de materiales: cantidades físicas

- Pruebas de materiales: pruebas no destructivas

7.2.3. Para el caso de los laboratorios de calibración deberán participar como mínimo una vez cada cuatro años en pruebas de aptitud u otras comparaciones para cada magnitud a calibrar o medir (ejemplo: voltaje, masa, temperatura etc.); correspondientes al alcance de la Acreditación del laboratorio".

Esta comparación siguió los lineamientos de la INTE ISO/IEC Guía 43-1:2000. "Ensayo de aptitud por comparaciones interlaboratorios. Parte 1: Desarrollo y funcionamiento de programas de ensayos de aptitud".

"El seguimiento debe ser planificado y revisado, y puede incluir los siguientes elementos: 
- materiales de referencia secundarios;

- la participación en comparaciones interlaboratorios o programas de ensayos de aptitud;

- la repetición de ensayos o calibraciones utilizando el mismo método o métodos diferentes;

- la repetición del ensayo o de la calibración de los objetos retenidos;

- la correlación de los resultados para diferentes características de un ítem."

Para una mejor comprensión se incluyen las siguientes definiciones. También se puede consultar el VIM (Vocabulario Internacional de Metrología).

- A CA: Amperios corriente alterna.

- A CD: Amperios corriente directa.

- CAMET: subregión metrológica centroamericana, del Sistema Interamericano de Metrología.

- Comparación interlaboratorio: organización, realización y evaluación de ensayos o calibraciones sobre el mismo objeto de ensayo o sobre objetos de ensayos similares, por dos o más laboratorios, de acuerdo con condiciones predeterminadas.

- ECA: Ente Costarricense de Acreditación.

- ICE: Instituto Costarricense de Electricidad.

- Incertidumbre estándar: incertidumbre del resultado de una medición expresada como una desviación estándar.

- Incertidumbre estándar combinada: incertidumbre estándar del resultado de una medición cuando el resultado se obtiene a partir de los valores de algunas otras magnitudes, igual a la raíz cuadrada positiva de una suma de términos, siendo estos términos las varianzas y covarianzas de estas otras magnitudes ponderadas de acuerdo a cómo el resultado de la medición varía con respecto a cambios en estas magnitudes.

- Incertidumbre expandida: cantidad que define un intervalo alrededor de una medición del que se puede esperar que abarque una fracción grande de la distribución de valores que razonablemente pudieran ser atribuidos al mensurando.
- INTECO: Instituto Nacional de Normas Técnicas de Costa Rica.

- LACOMET: Laboratorio Costarricense de Metrología.

- LMVE: Laboratorio Metrológico de Variables Eléctricas.

- Medición de periodo: medición del intervalo de tiempo entre dos sucesos, el primer suceso marcará el instante del comienzo (arranque) del intervalo de tiempo, marcando el segundo suceso el instante de finalización (parada) de la cuenta.

- Método de estrella: Es cuando el instrumento o material de referencia utilizado en la comparación es devuelto al laboratorio de referencia cada vez que el participante finaliza la medición.

- $\Omega$ : ohm.

- Patrón viajero: Patrón, algunas veces de construcción especial, para ser transportado entre diferentes lugares.

- V CA: Voltaje corriente alterna.

- V CD: Voltaje corriente directa.

\section{METODOLOGÍA}

La comparación de calibración en multímetros se realizó con el fin de estimar los niveles de concordancia en la calibración de voltaje, corriente y resistencia entre los laboratorios participantes, incluyendo la desviación y la incertidumbre de medición asociada. El requisito para participar fue tener la capacidad para calibrar multímetros digitales.

Las fechas de participación fueron desde el 24 de abril del 2009 hasta el 17 de setiembre del 2009. Los laboratorios participantes fueron doce, el laboratorio piloto y once más, codificados con los números del 1 al 12, en orden aleatorio.

Laboratorio piloto:

Laboratorio Metrológico de Variables Eléctricas (ICE-LMVE)

Instituto Costarricense de Electricidad

Correo electrónico:1mve@ice.go.cr 
Cuadro 1. Valores para la calibraciones del multímetro.

\begin{tabular}{ccccc}
\hline Punto & Unidades & Escala & Valor nominal & Frecuencia \\
\hline 1 & $\mathrm{mV}$ & 300 & 100 & \\
2 & $\mathrm{~V}$ & 3 & 1 & \\
3 & $\mathrm{~V}$ & 30 & 10 & \\
4 & $\mathrm{~mA}$ & 300 & 100 & \\
5 & $\mathrm{~mA}$ & 1000 & 1000 & \\
6 & $\mathrm{~V}$ & 300 & 100 & $@ 60 \mathrm{~Hz}$ \\
7 & $\mathrm{~V}$ & 300 & 120 & $@ 60 \mathrm{~Hz}$ \\
8 & $\mathrm{~mA}$ & 1000 & 500 & $@ 1 \mathrm{kHz}$ \\
9 & $\mathrm{~mA}$ & 300 & 10 & $@ 5 \mathrm{kHz}$ \\
10 & $\mathrm{~mA}$ & 300 & 100 & $@ 5 \mathrm{kHz}$ \\
11 & $\mathrm{~mA}$ & 1000 & 1000 & $@ 5 \mathrm{kHz}$ \\
12 & $\mathrm{~mA}$ & 1000 & 1000 & $@ 60 \mathrm{~Hz}$ \\
13 & $\mathrm{k} \Omega$ & 3 & 1 & \\
14 & $\mathrm{k} \Omega$ & 30 & 10 & \\
15 & $\mathrm{k} \Omega$ & 300 & 100 & \\
16 & $\mathrm{k} \Omega$ & 3000 & 1000 & \\
\hline
\end{tabular}

Fuente: (LMVE, 2009).

Laboratorios participantes:

- Laboratorio Metrológico de Variables Eléctricas (LMVE), Instituto Costarricense de Electricidad.

- Laboratorio de Metrología de DT DEP (LAMETRO), Instituto Costarricense de Electricidad.

- Metcal Engineering Services.

- SCM Metrología y Laboratorios S. A.

- Laboratorio de Calibración de Equipos de Medición de Bridgestone de Costa Rica.

- Calibraciones de América CAMÉRICAS.A.

- UEN CENCE, Instituto Costarricense de Electricidad.

- LMVE-DTOM, Instituto Costarricense de Electricidad.

- DIM Metrología.

- Centro Nacional de Metrología de Panamá (CENAMEP)

- A Uno Calibration Laboratory S.A.
- LMVE-CSTRC, Instituto Costarricense de Electricidad.

Se utilizó como patrón viajero un multímetro marca Hioki, modelo 3235, propiedad del ICE. Fue responsabilidad de cada participante el transporte desde y hacia el LMVE del patrón viajero. Cada participante entregó al LMVE el formulario de recepción y de compromiso de devolución en la fecha acordada. Cada participante debía informar al LMVE con un día de anticipación sobre la fecha de devolución al LMVE. El patrón viajero fue transportado en una caja protectora. Cada participante usó sus procedimientos para estas actividades.

Estaba previsto que en caso de falla el participante debía informar lo antes posible al LMVE para coordinar las mejores actividades a realizar; afortunadamente, no hubo fallas del patrón durante la actividad. Cada participante cubrió el costo del transporte del patrón viajero 
desde y hacia el LMVE. Fue recomendada una póliza temporal de seguro o cualquier cobertura que el participante pudiera disponer.

Se usó el método de estrella para la circulación del patrón, estando una semana en el sitio de cada participante y una semana en el LMVE, donde se realizaron mediciones previas de caracterización $\mathrm{y}$ se realizaron mediciones intercaladas entre cada participante.

Los valores correspondientes a V CD, V CA, A CD, A CA, $\Omega$, fueron escogidos de una lista de sesenta y seis posibles.

\subsection{Incertidumbre de la medición}

Los participantes reportaron sus resultados con la incertidumbre asociada, con un factor de cobertura de dos sigma $(95,45 \%)$, considerando como mínimo las incertidumbres debidas a la especificación del patrón del participante, resolución del patrón viajero y la variabilidad de las mediciones repetidas.

\subsection{Reporte de mediciones}

Para el reporte de las mediciones, cada participante, usando sus propios formatos, envió al LMVE sus resultados de medición.

Nota: Cada participante reportó sus resultados de acuerdo con sus procedimientos, sin que le correspondiera al laboratorio piloto la evaluación de su calidad técnica.

\subsection{Valor de referencia}

El valor de referencia de la comparación fue definido por el LMVE como el promedio de las mediciones realizadas en el patrón viajero antes y después de las mediciones de cada participante, usando un calibrador multifunción Fluke 5720A, con trazabilidad al SI mediante los certificados de calibración ICE-LMVE-I-2077-31jul2008 y ICE-LMVE-I-2077-02set2009. El patrón viajero mantuvo estabilidad en sus mediciones, dentro de sus especificaciones, durante toda la comparación.

\section{RESULTADOS DE LA COMPARACIÓN}

En las Figuras de la 1 a la 16 se grafican los límites superior e inferior de incertidumbre del LMVE al 95,45 \% de confianza, en sus respectivas unidades. El valor medido reportado por el LMVE es la línea continua que se encuentra entre las líneas discontinuas. Los datos fueron graficados con la cantidad de dígitos reportados por cada participante, lo cual muestra una variación referente al informe expuesto en los Cuadros 2 y 3. Los datos fueron truncados a la cantidad de dígitos correspondientes a la resolución del instrumento.

Cada punto representa el valor reportado por cada participante en cada medición, con su respectiva incertidumbre. La información brindada en los cuadros y en las figuras es suficiente para que cada participante realice su cálculo de error normalizado, de acuerdo con las referencias INTECO y ECA. De estos datos se puede ver el error que tiene cada participante en su sistema de calibración y su incertidumbre total. Esta información es útil para que los usuarios de servicios de calibración puedan ver el desempeño de sus proveedores, para que los organismos que proveen acreditación evalúen a sus acreditados y para que los propios laboratorios analicen y mejoren sus procesos de calibración. Los resultados de esta comparación interlaboratorio son de utilidad para que cada laboratorio respalde sus capacidades de medición y calibración, de acuerdo con la norma de competencia técnica de laboratorios y la guía para ensayos de aptitud por comparación interlaboratorios.

Nota: Los participantes cuatro y once no aparecen en los Cuadros ni en las figuras, debido a que uno de ellos no entregó resultados y el otro solicitó que sus datos no fueran publicados. Los participantes dos y siete no reportaron algunos valores de corriente, como se muestra en los Cuadros 3 y 4.

En esta comparación participó, además de los laboratorios costarricenses, el laboratorio nacional de Panamá, CENAMEP. Se espera que esta iniciativa se continúe extendiendo con la participación de otros laboratorios de CAMET, para el beneficio del movimiento de servicios y productos y la mejora en la calidad de vida de los habitantes, por medio de mejora en la capacidad metrológica de nuestros países. 


\subsection{Datos de mediciones}

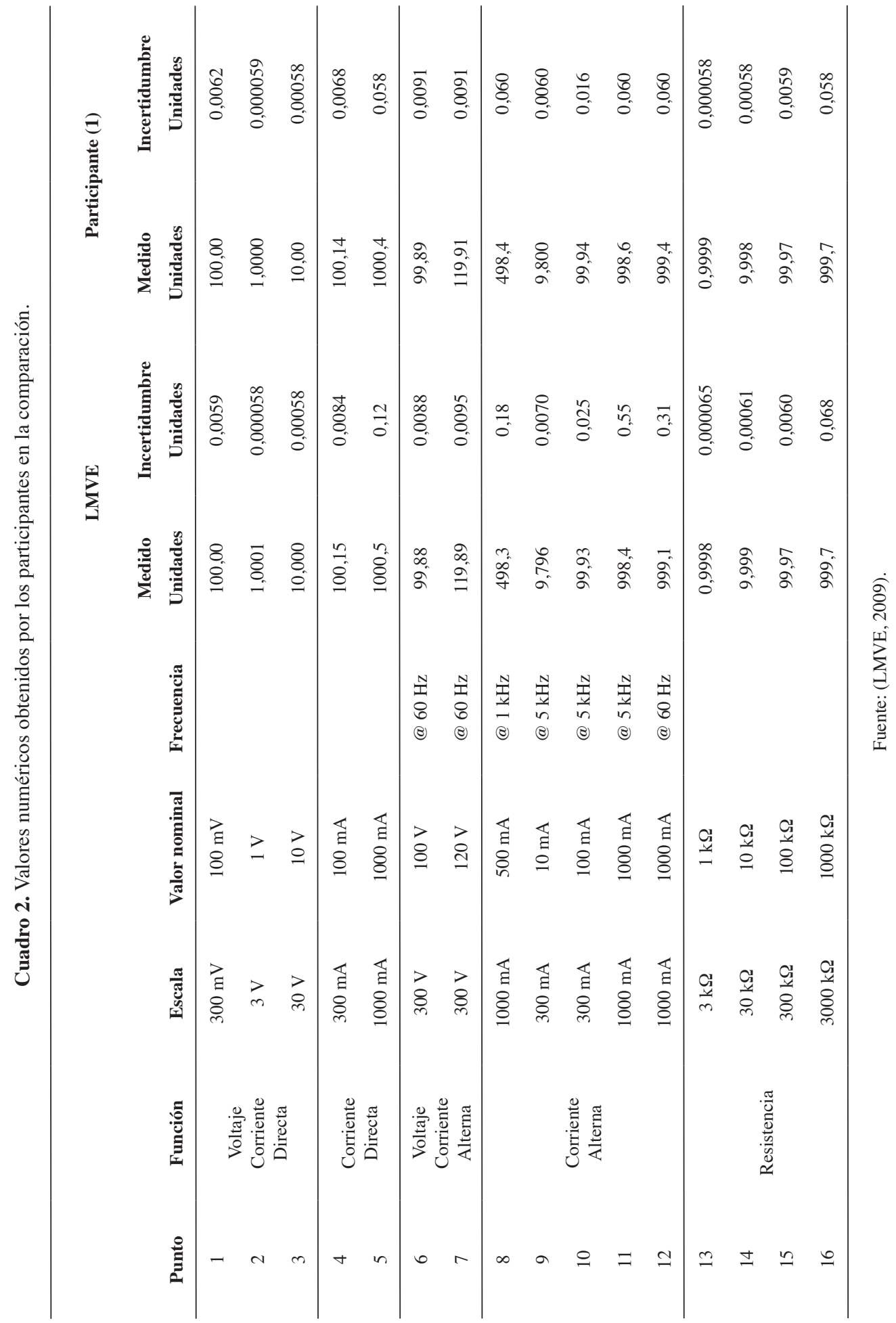




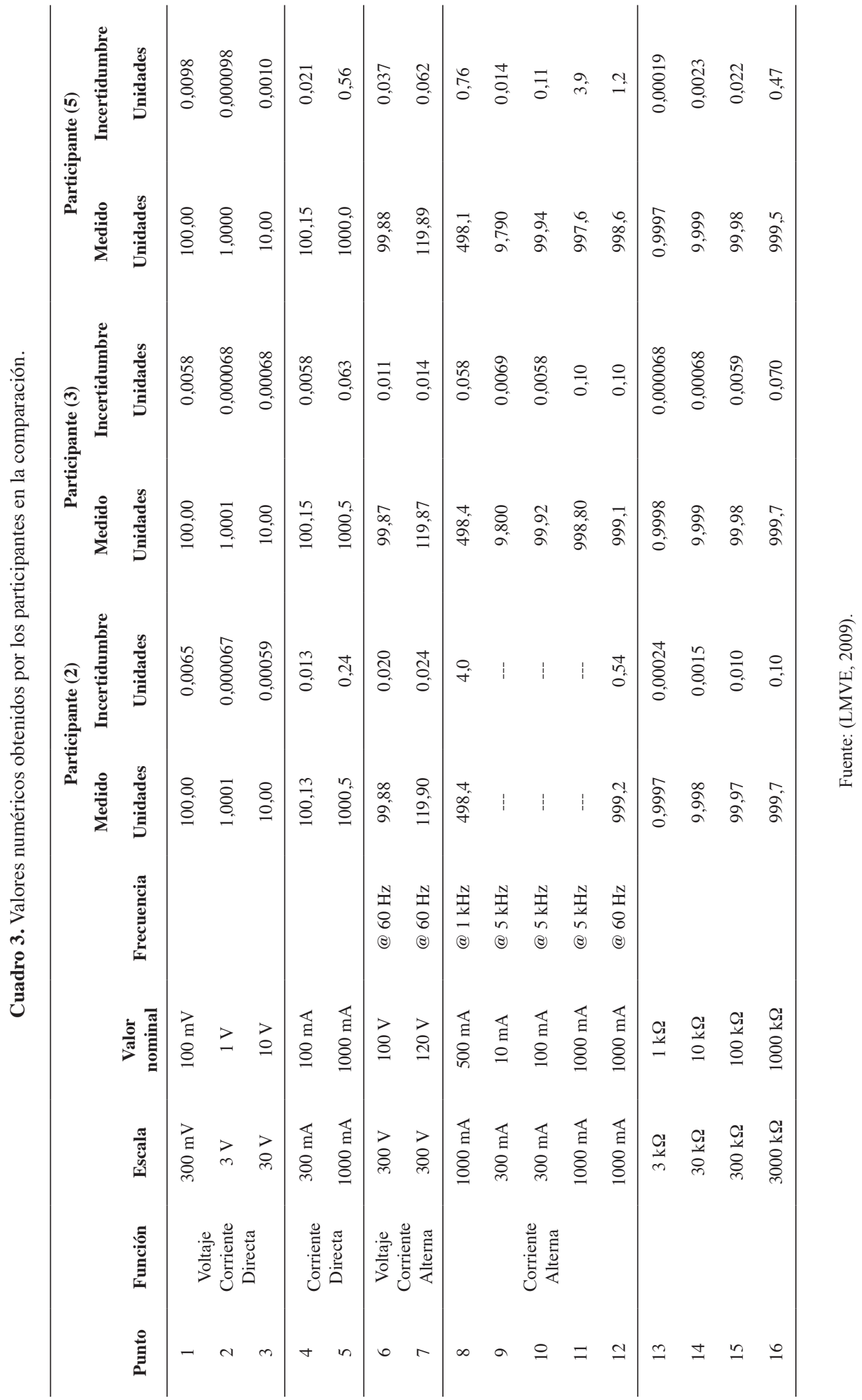




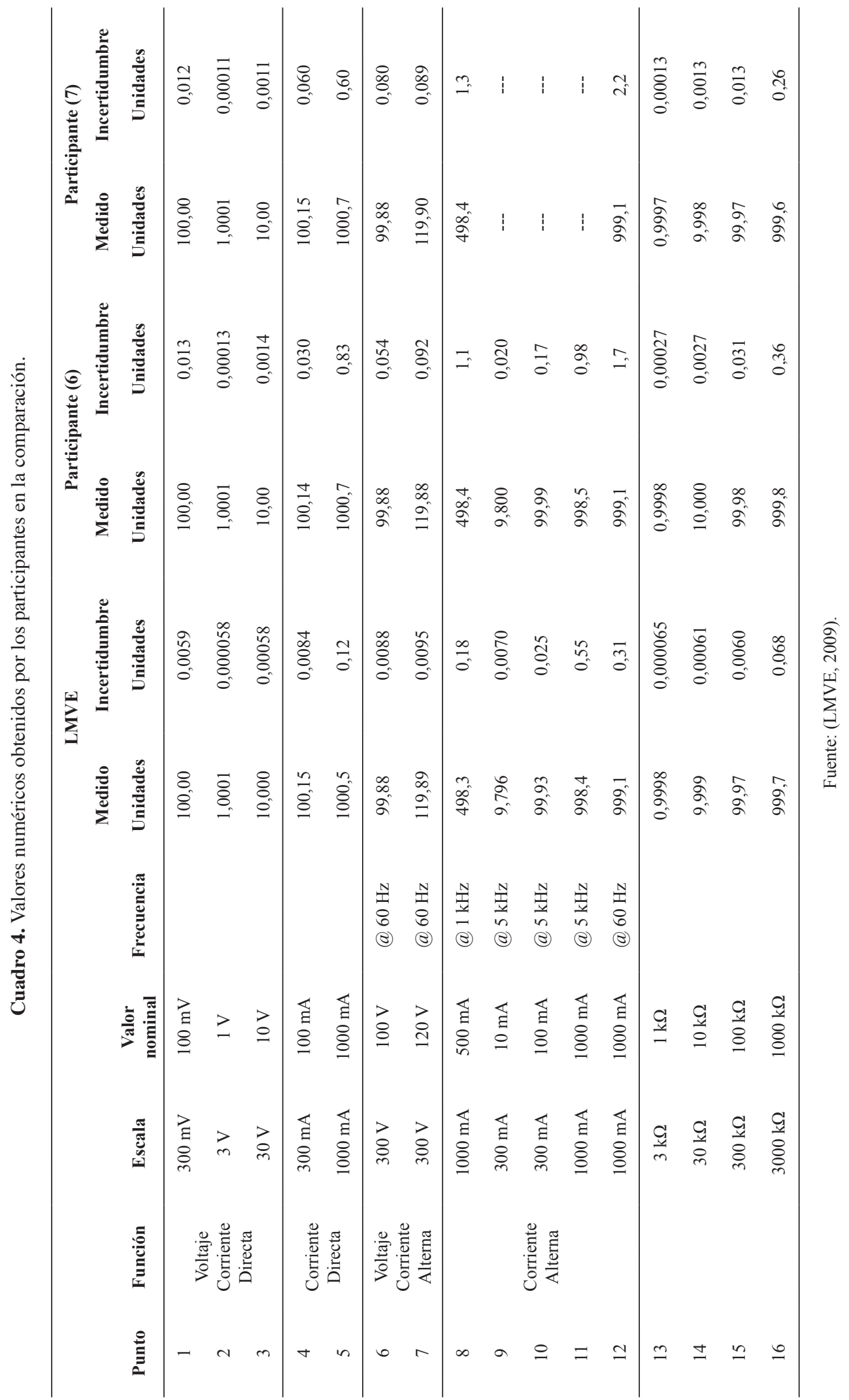




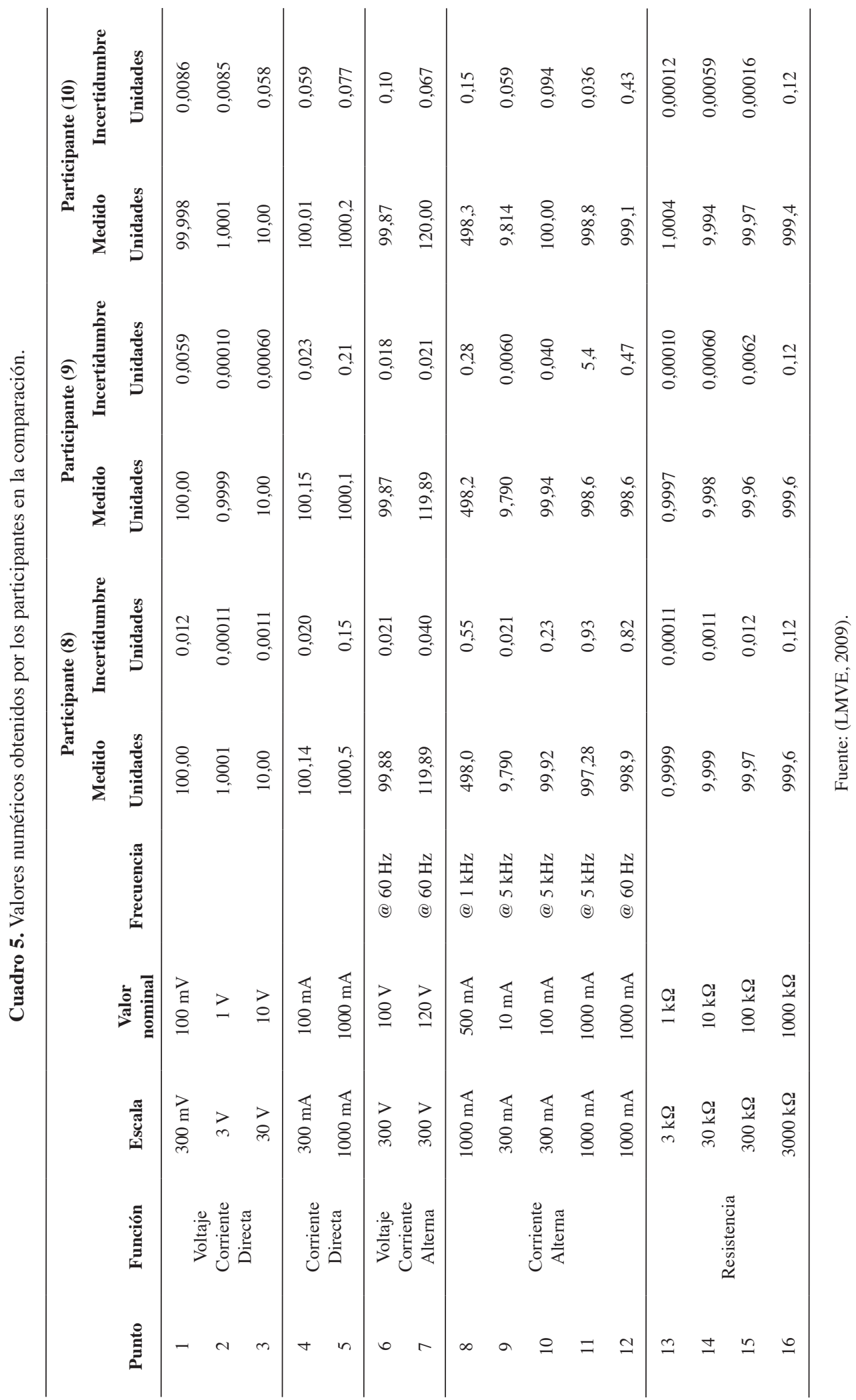




\subsection{Resultados de la intercomparaciòn}

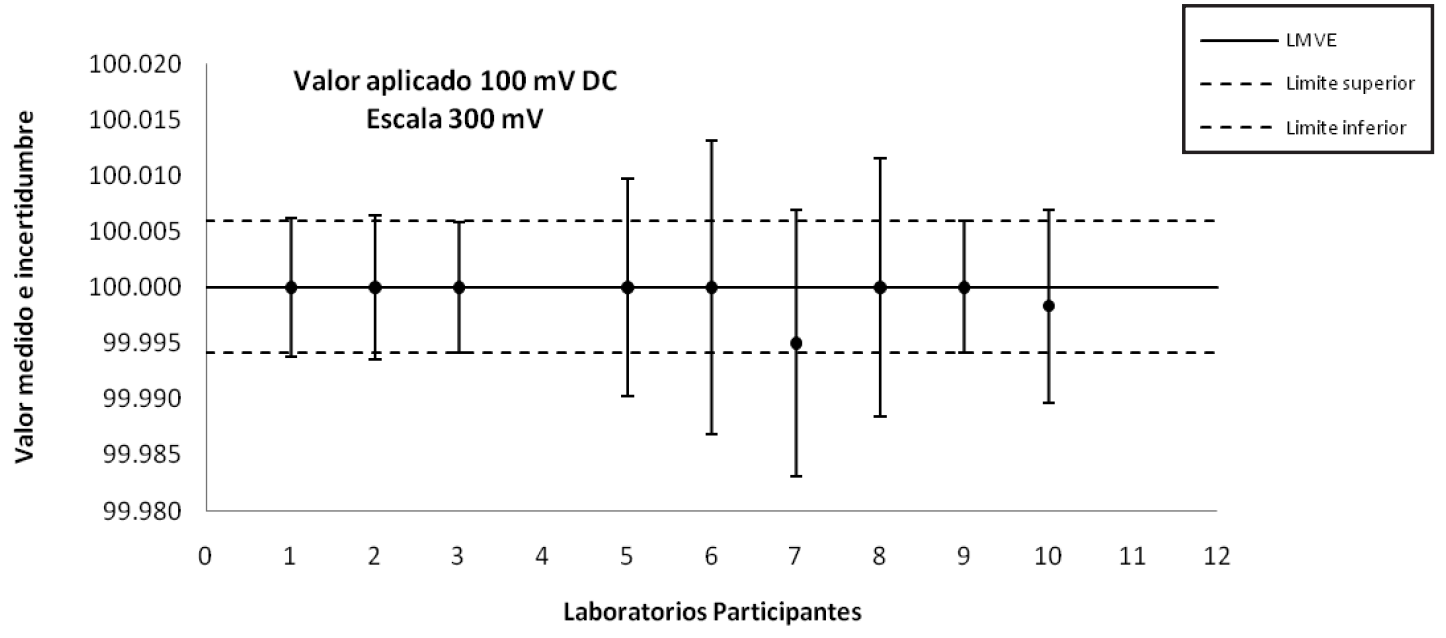

Figura 1. Gráfico de resultados de la comparación en 100 mV.

Fuente: (LMVE, 2009)

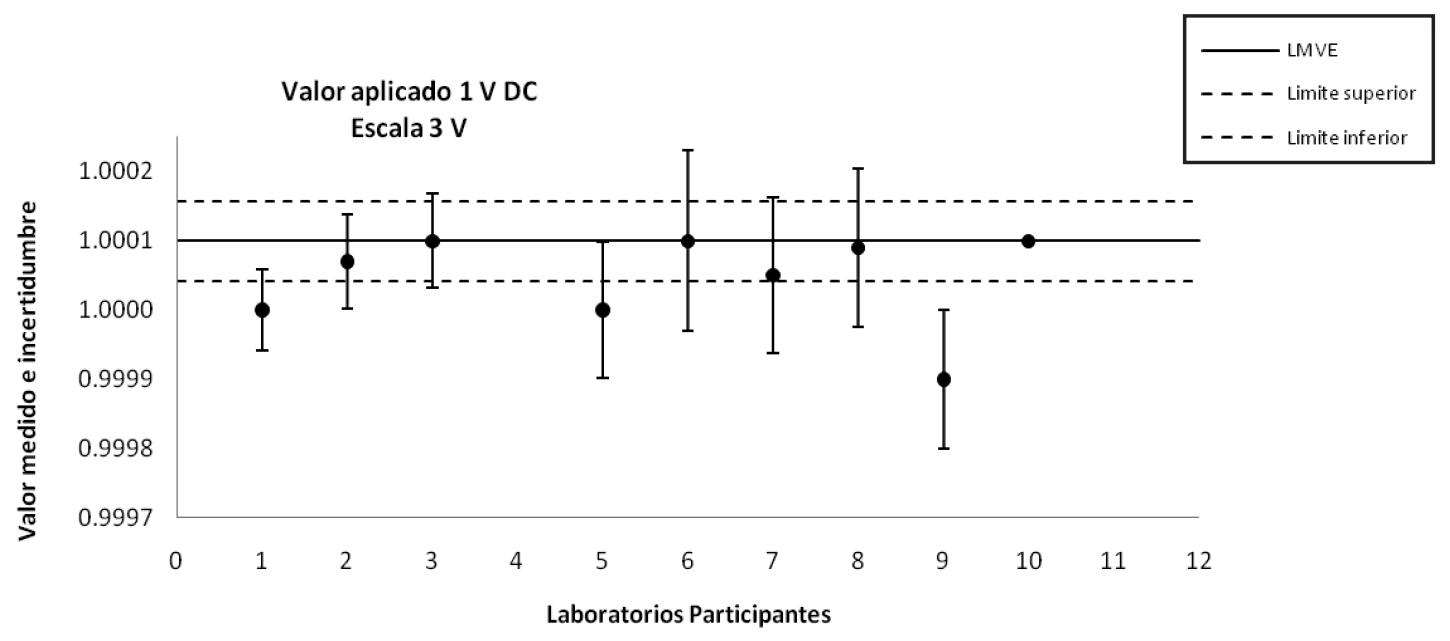

Nota: La incertidumbre reportada por el participante (10) no se incluye en el gráfico porque excede la coordenada vertical

Figura 2. Gráfico de resultados de la comparación en $1 \mathrm{~V}$.

Fuente: (LMVE, 2009). 


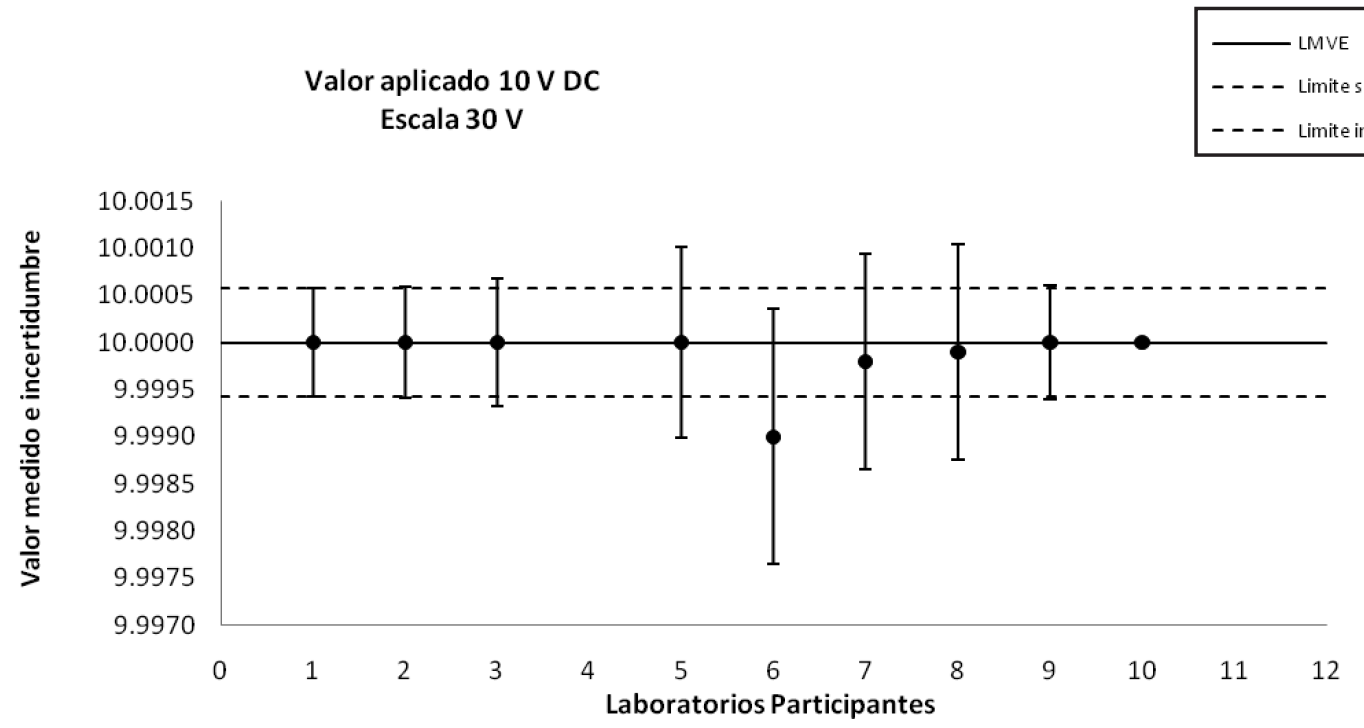

Figura 3. Gráfico de resultados de la comparación en $10 \mathrm{~V}$.

Fuente: (LMVE, 2009).

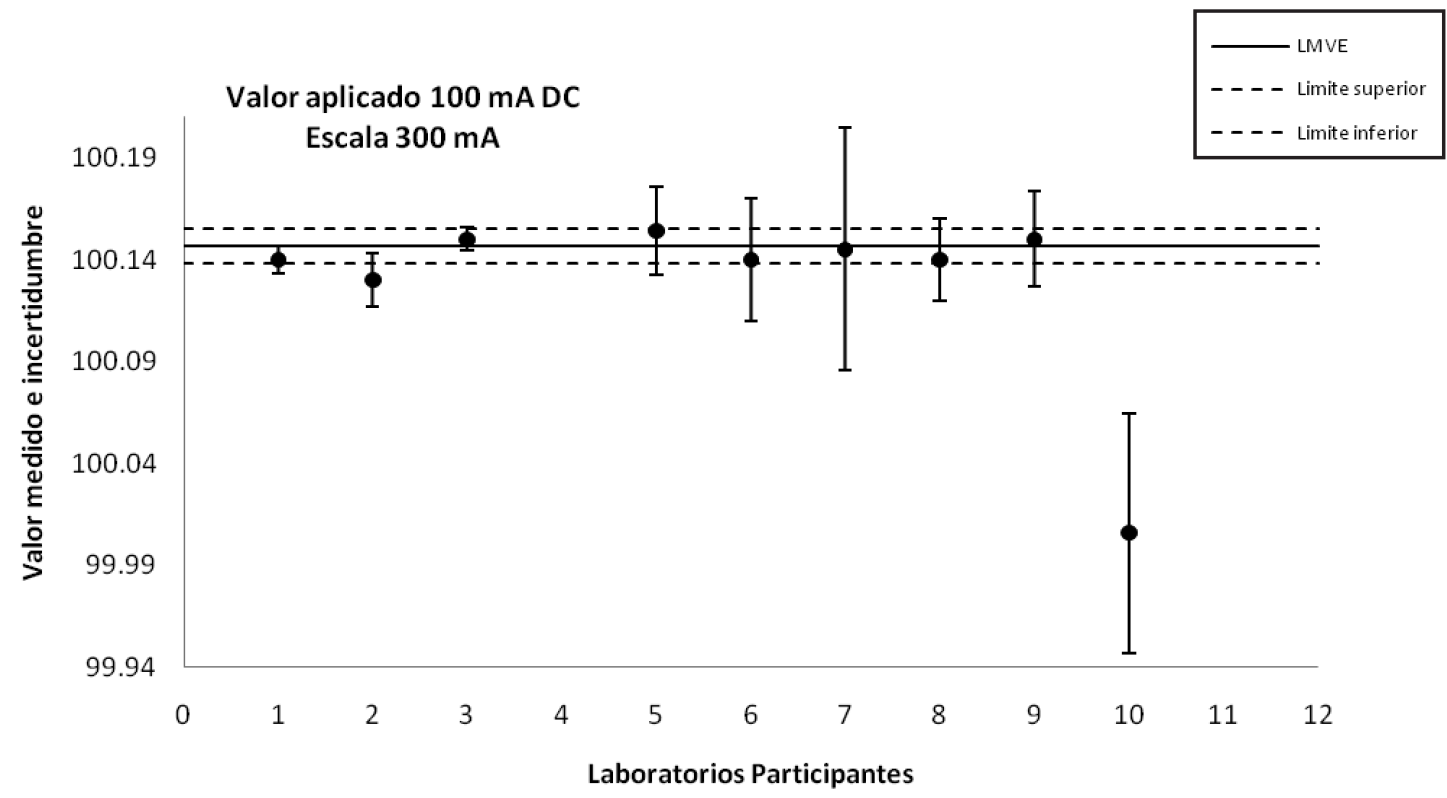

Figura 4. Gráfico de resultados de la comparación en 100 mA DC.

Fuente: (LMVE, 2009). 


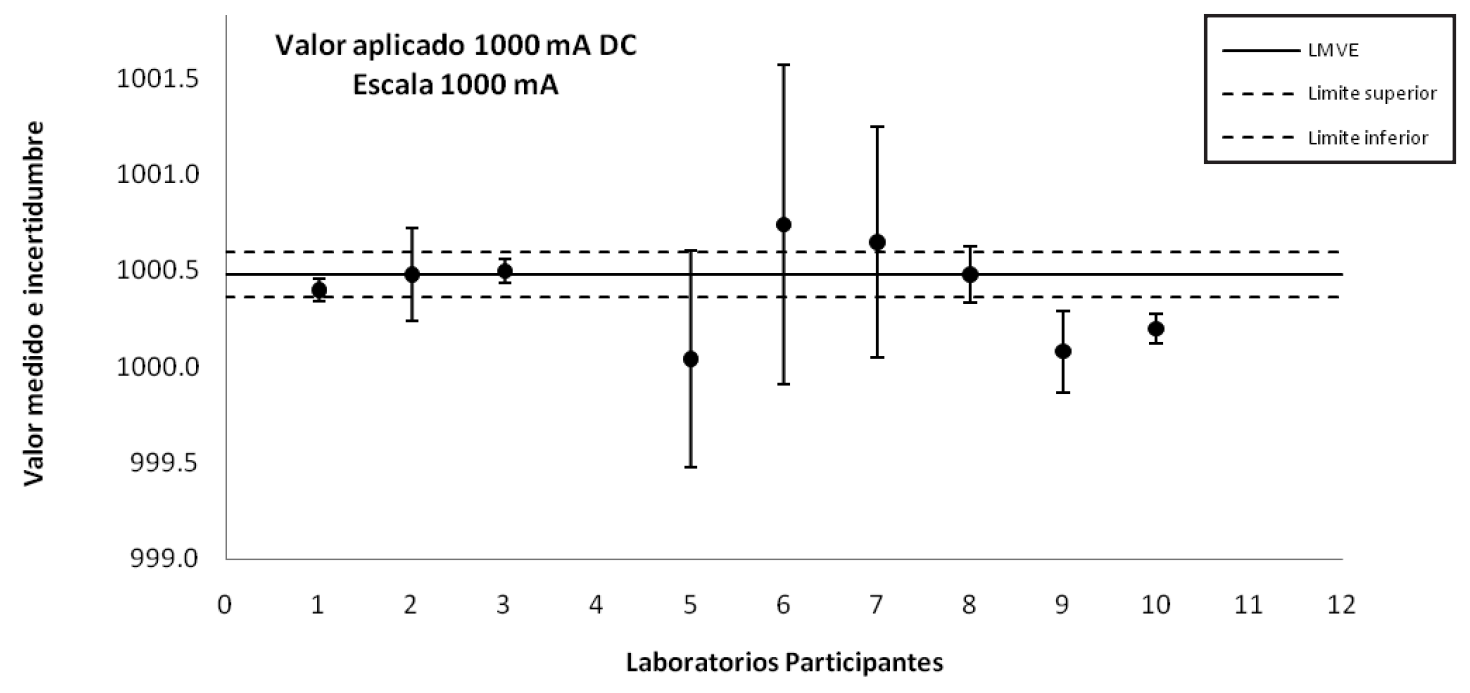

Figura 5. Gráfico de resultados de la comparación en 1000 mA DC.

Fuente: (LMVE, 2009).

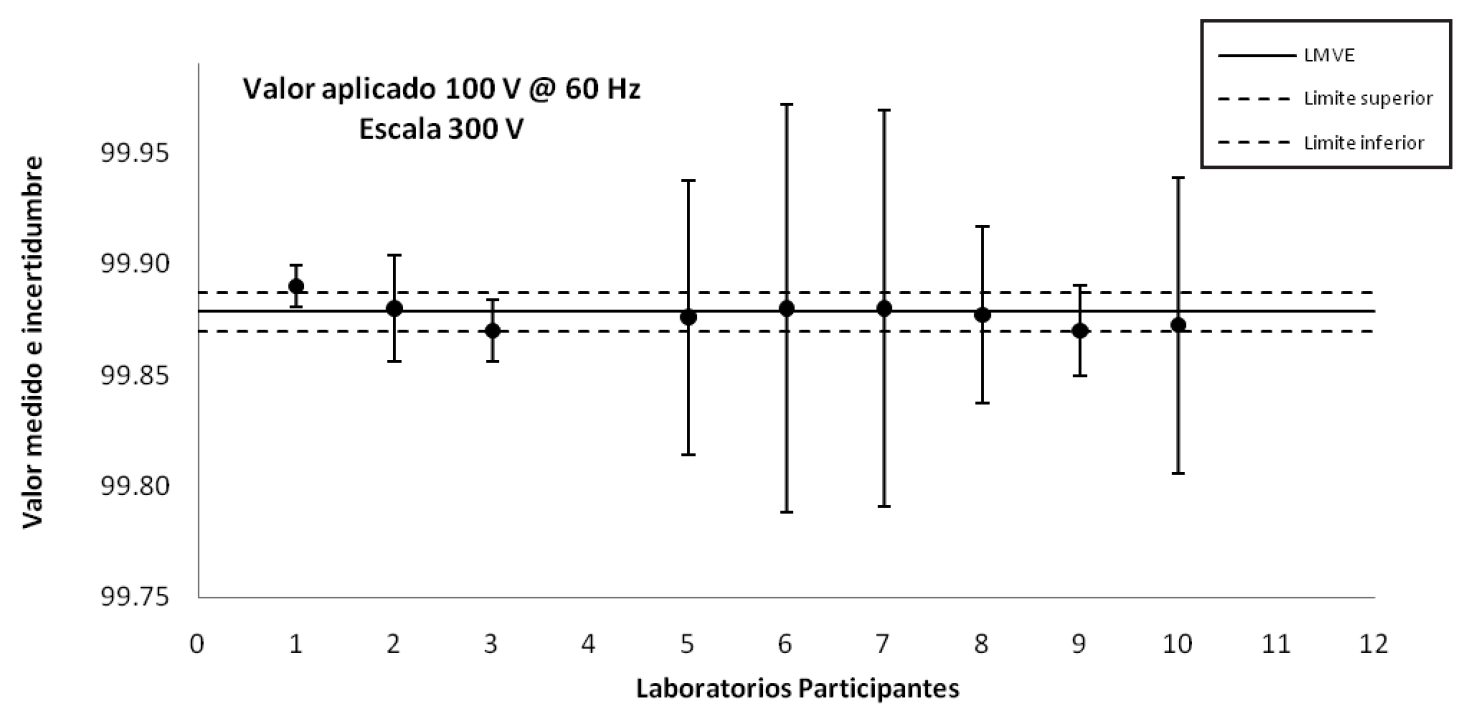

Figura 6. Gráfico de resultados de la comparación en 100 VCA @ 60 Hz.

Fuente: (LMVE, 2009). 


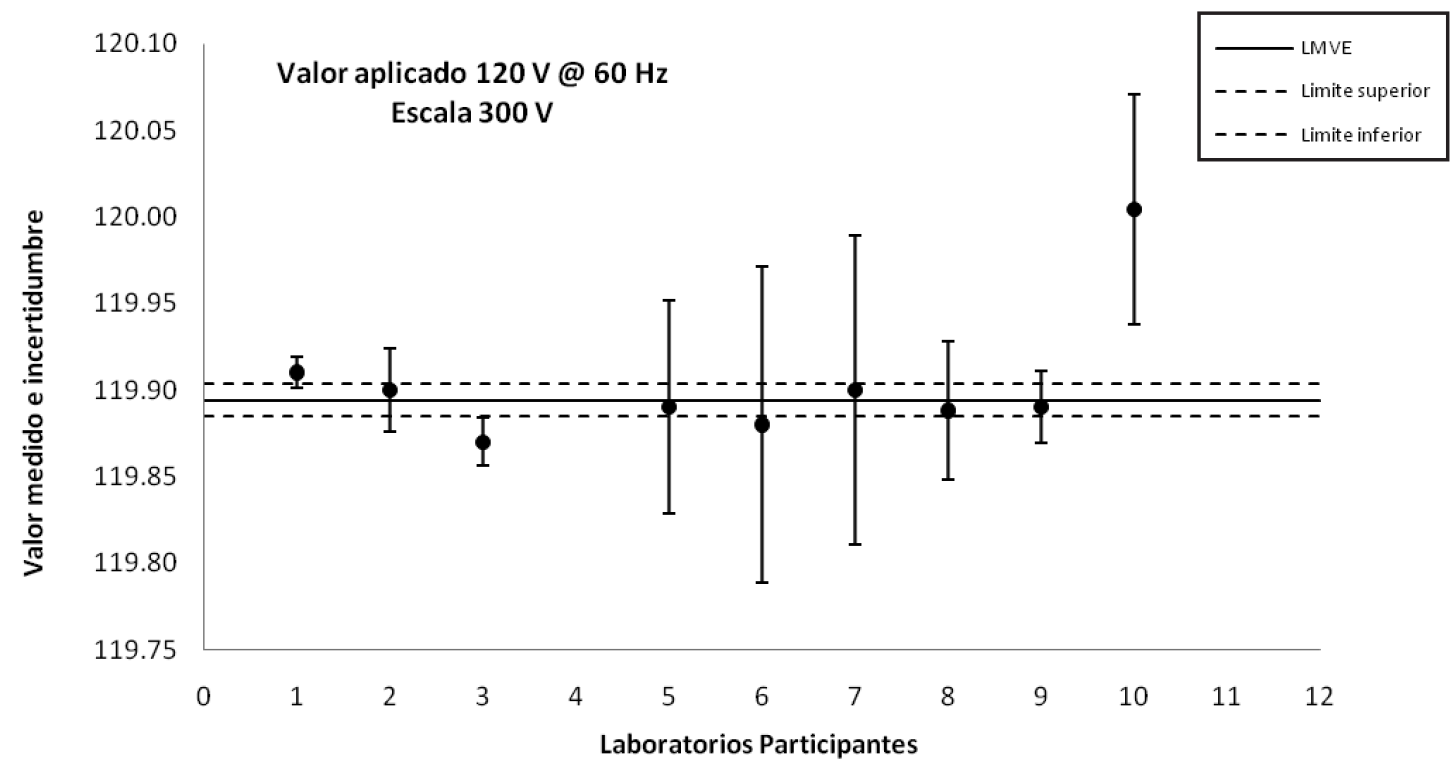

Figura 7. Gráfico de resultados de la comparación en 120 VCA @ 60 Hz.

Fuente: (LMVE, 2009).

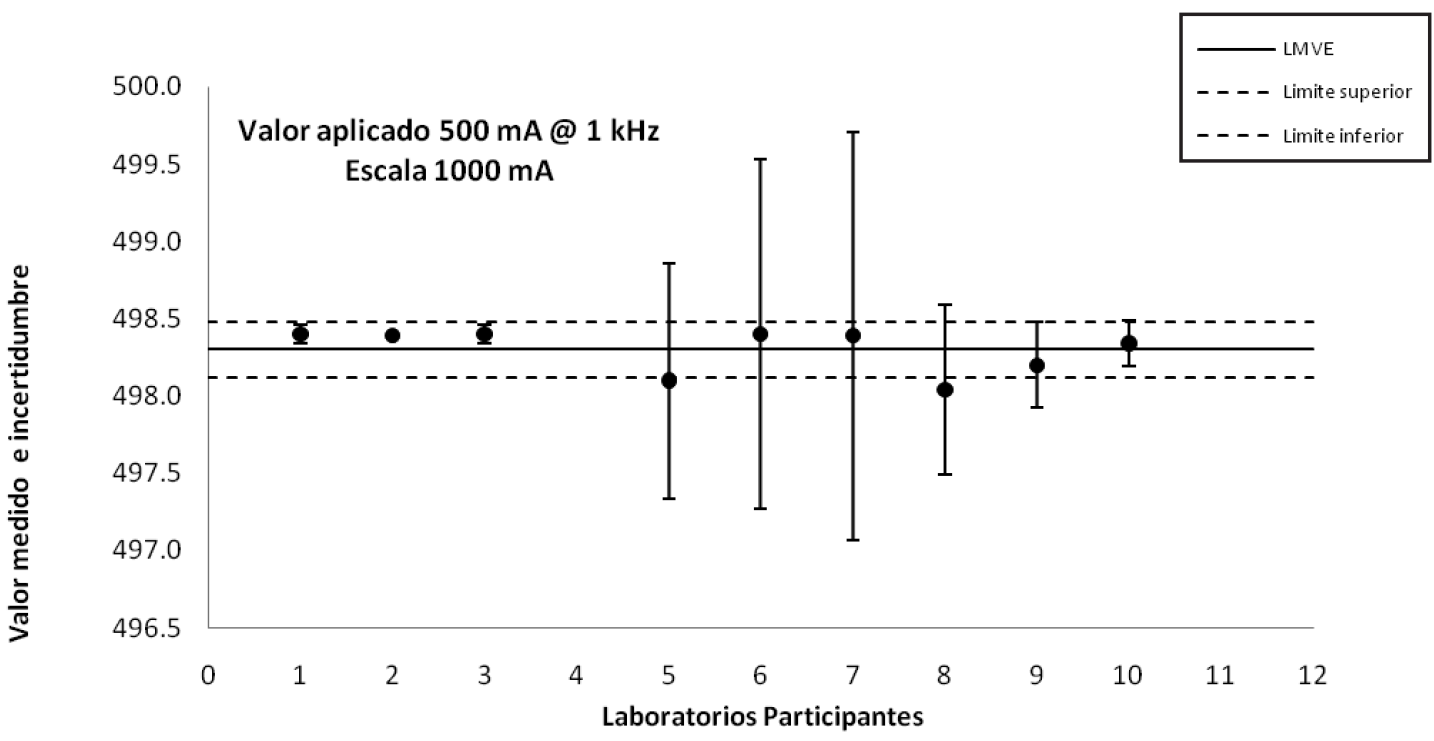

Nota: La incertidumbre reportada por el participante (2) no se incluye en el gráfico porque excede la coordenada vertical.

Figura 8. Gráfico de resultados de la comparación en 500 mA CA @ 1 kHz.

Fuente: (LMVE, 2009). 


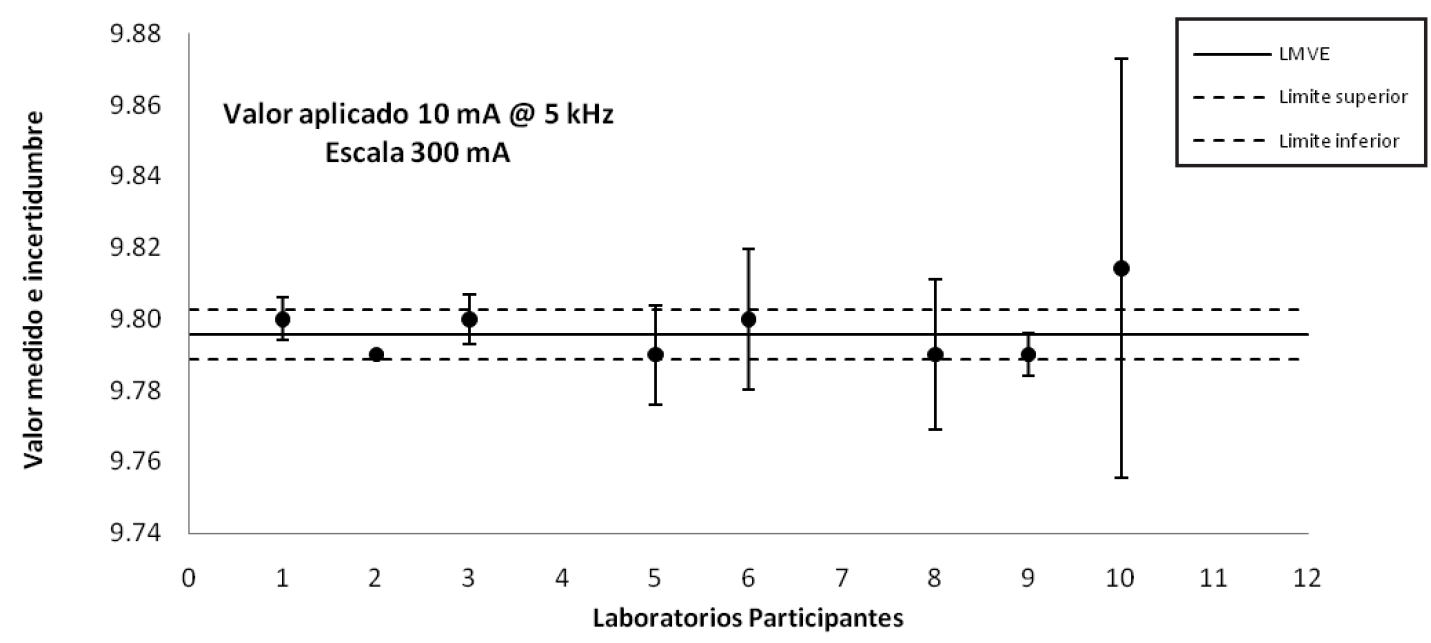

Nota: La incertidumbre reportada por el participante (2) no se incluye en el gráfico porque excede la coordenada vertical.

Figura 9. Gráfico de resultados de la comparación en 10 mA CA @ 1 kHz.

Fuente: (LMVE, 2009).

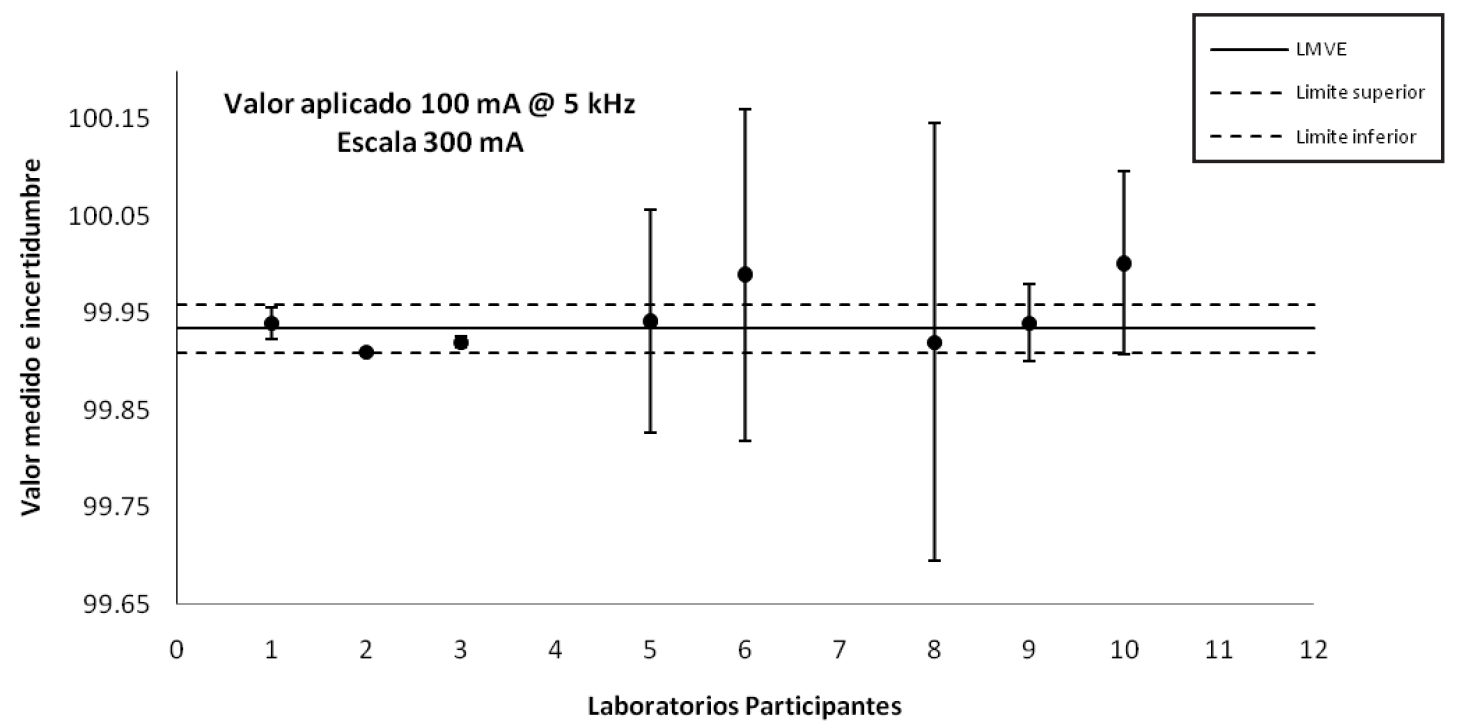

Nota: La incertidumbre reportada por el participante (2) no se incluye en el gráfico porque excede la coordenada vertical.

Figura 10. Gráfico de resultados de la comparación en 100 mA CA @ 1 kHz.

Fuente: (LMVE, 2009). 


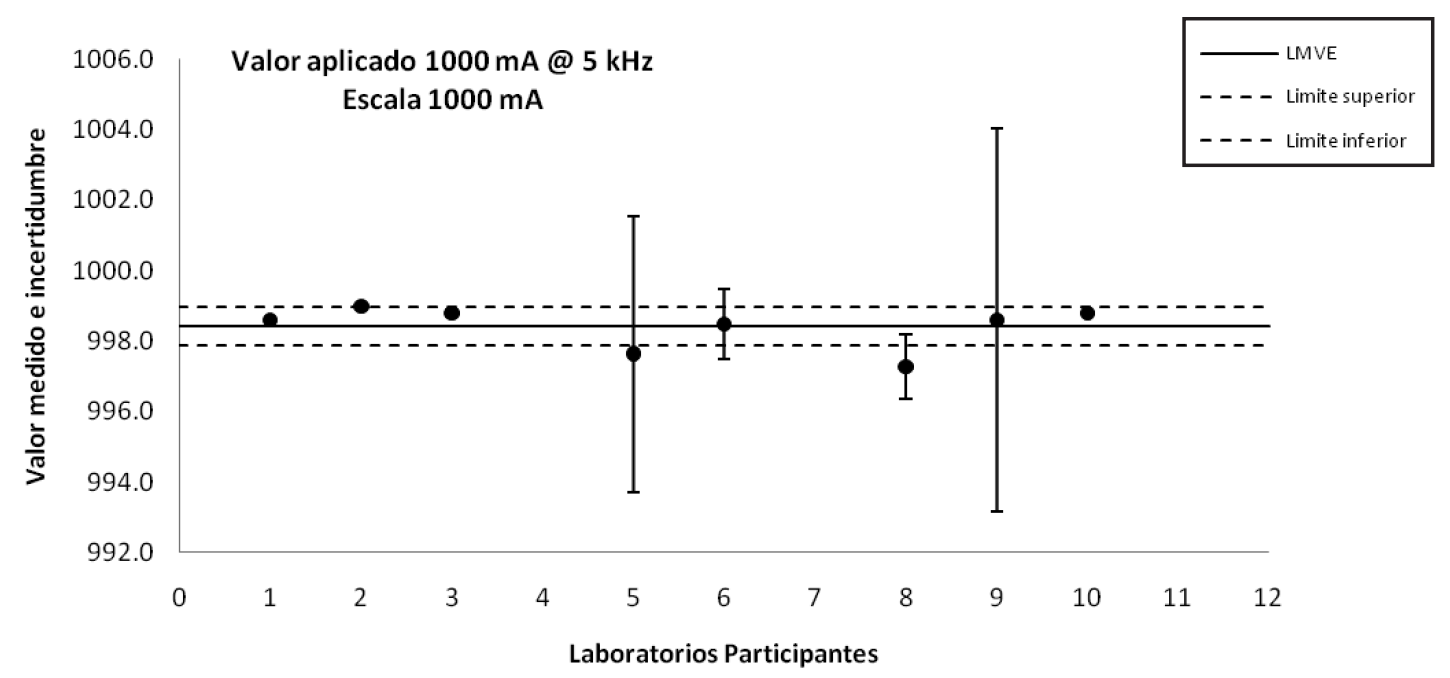

Nota 1: La incertidumbre reportada por el participante (2) no se incluye en el gráfico porque excede la coordenada vertical.

Nota 2: Las incertidumbres de los participantes (1, 3 y 10) están incluidas pero su aporte es insignificante y no es posible apreciarlas bien en el gráfico.

Figura 11. Gráfico de resultados de la comparación en 1000 mA CA @ 1 kHz.

Fuente: (LMVE, 2009).

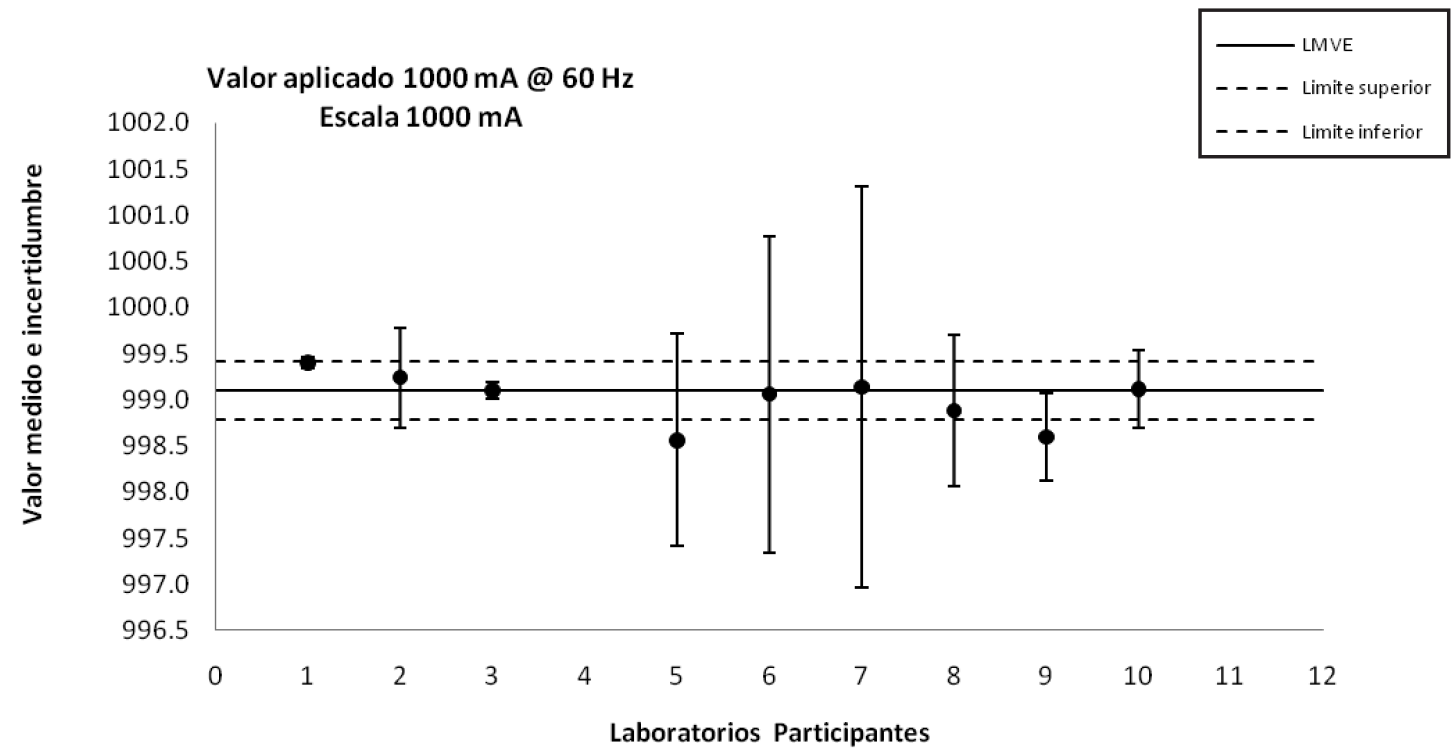

Figura 12. Gráfico de resultados de la comparación en 1000 mA CA @ 60 Hz.

Fuente: (LMVE, 2009). 


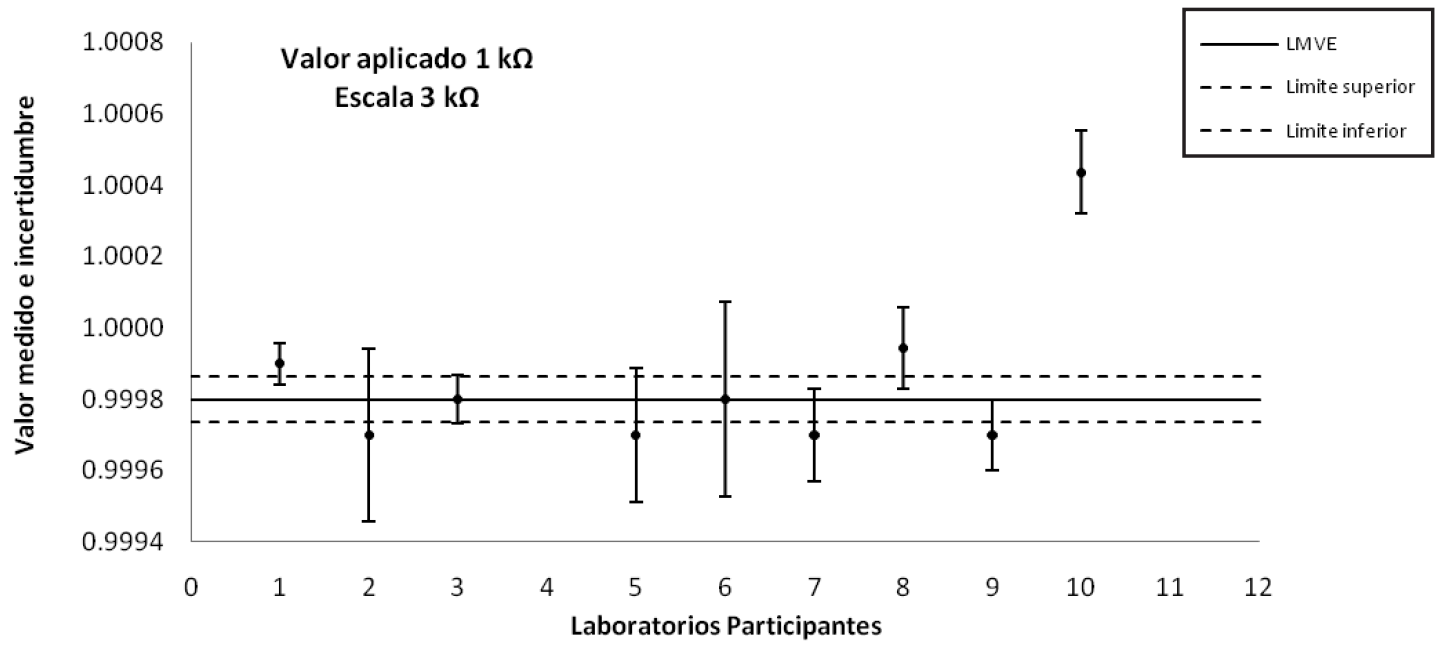

Figura 13. Gráfico de resultados de la comparación en $1 \mathrm{k} \Omega$.

Fuente: (LMVE, 2009).

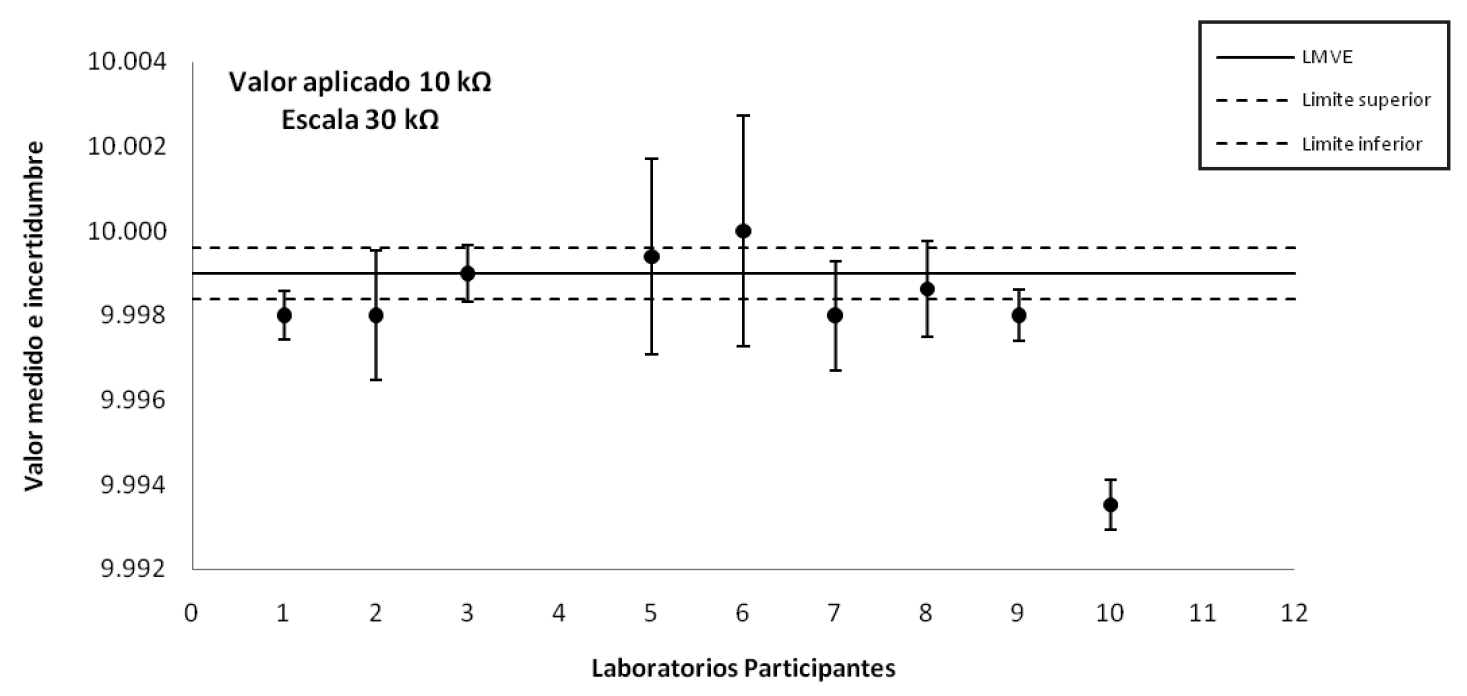

Figura 14. Gráfico de resultados de la comparación en $10 \mathrm{k} \Omega$.

Fuente: (LMVE, 2009). 


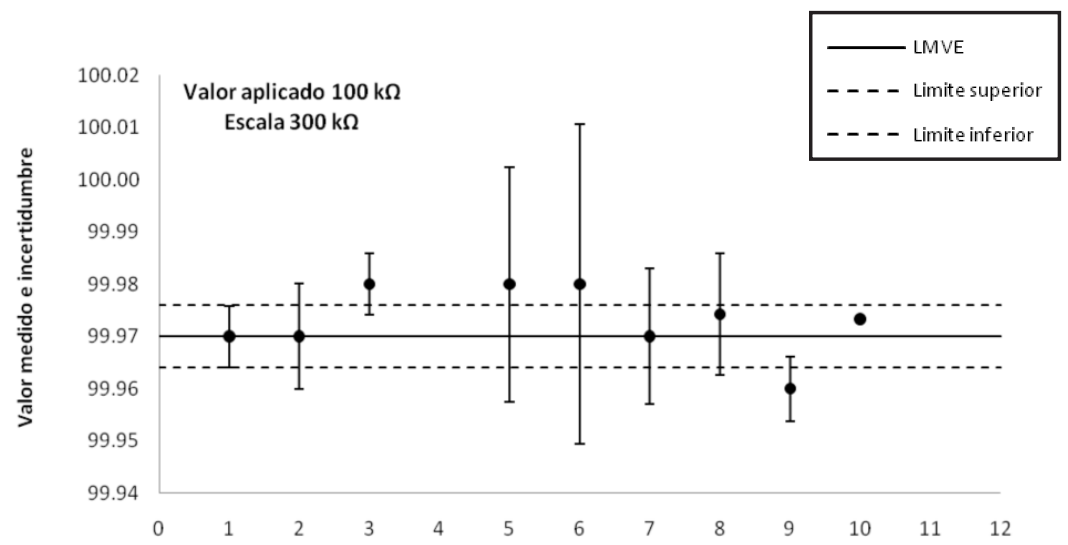

Nota: La incertidumbre del participante (10) está incluida pero su aporte es insignificante y no es posible apreciarla bien en el gráfico.

Figura 15. Gráfico de resultados de la comparación en $100 \mathrm{k} \Omega$.

Fuente: (LMVE, 2009).

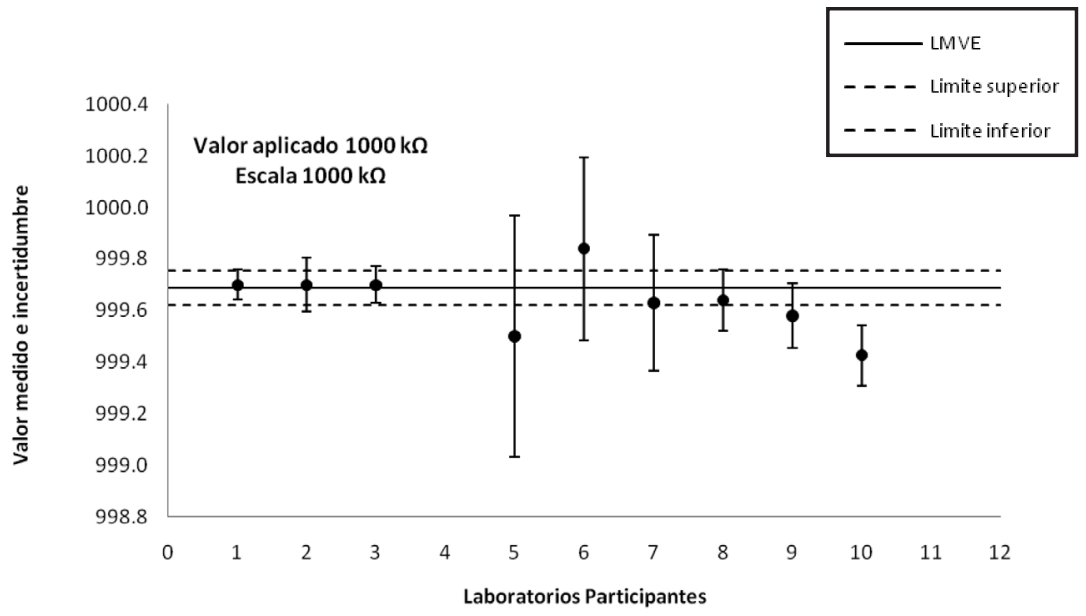

Figura 16. Gráfico de resultados de la comparación en $1000 \mathrm{k} \Omega$.

Fuente: (LMVE, 2009). 


\section{CONCLUSIONES}

1. La presentación de los datos de esta comparación en forma tabulada y gráfica sirve de referencia para la mejora de los laboratorios participantes.

2. Algunos participantes reportaron incertidumbres iguales o menores que el laboratorio piloto. Estos participantes deben revisar sus cálculos de incertidumbre para comprobar si están subestimando su incertidumbre.

3. Las diferencias en los valores medidos y en las incertidumbres pueden dar a los clientes de los laboratorios participantes una indicación de que deben comparar sus servicios y pedirles mejora en su calidad.

4. Los laboratorios cuyas desviaciones e incertidumbres no son congruentes con los valores de referencia deben realizar acciones de mejora, de acuerdo con la política y criterios para la participación en ensayos/pruebas de aptitud y otras comparaciones para los laboratorios, del Ente Costarricense de Acreditación (www. eca.or.cr).

\section{REFERENCIAS BIBLIOGRÁFICAS}

ECA (Ente Costarricense de Acreditación). (2006). ECA-MC-P17 Política y criterios para la participación en ensayos/pruebas de aptitud y otras comparaciones para los laboratorios. Versión 1. Ente Costarricense de Acreditación, San José: Editor.

HIOKI E.E. (1994) Manual del usuario Digital hitester 3235. Japón.
INTECO (Instituto Costarricense de Normas Técnicas). (2005). INTE-ISO/IEC 17025:2005. Requisitos generales para la competencia de laboratorios de ensayo y calibración. (1a ed.) San José: Editor.

INTECO. (2000). INTE ISO/IEC Guía 431:2000. Ensayo de aptitud por comparaciones interlaboratorios. Parte 1: Desarrollo y funcionamiento de programas de ensayos de aptitud. San José: Editor.

JCGM 200:2008. Vocabulario internacional de metrología - Conceptos fundamentales y generales, y términos asociados (VIM). BIPM. Traducción al español del VIM $3^{\mathrm{a}}$. http://www.sim-metrologia.org.br/voca_int_ metro.pdf

\section{SOBRE LOS AUTORES}

\section{Juan Gómez Valverde}

Ingeniero Electricista, B. Sc.

Laboratorio Metrológico de Variables Eléctricas Instituto Costarricense de Electricidad

Teléfono: (506) 2283-6642

Facsímil: (506) 2234-8514

Apartado postal: 485-2050. San Pedro, Costa Rica. Correo electrónico: jgomezv@ice.go.cr

\section{Harold Sánchez Vargas}

Ingeniero de Sistemas Electrónicos, Ph. D. Laboratorio Metrológico de Variables Eléctricas Instituto Costarricense de Electricidad Teléfono: (506) 2283-4622

Facsímil: (506) 2234-8514

Apartado postal: 485-2050. San Pedro, Costa Rica. Correo electrónico: hsanchez@ice.go.cr 